\title{
Nasal Cavity and Paranasal Sinus Cancer by AJCC v8 Stage
}

National Cancer Institute

\section{Source}

National Cancer Institute. Nasal Cavity and Paranasal Sinus Cancer by A/CC v8 Stage. NCI

Thesaurus. Code C133074.

A term that refers to the staging of nasal cavity and paranasal sinus carcinoma according to the American Joint Committee on Cancer, 8th edition. 